\title{
NUMERICAL MODEL OF THE CYTOSKELETON STRUCTURATION DURING CELL SPREADING
}

\author{
Patrick Cañadas (1), Bernard Maurin (1), Haimad Baudriller (1), \\ Philippe Montcourrier (2), Nadir Bettache (2) \\ (1) Laboratoire de Mécanique et Génie Civil, UMR CNRS 5508, Université \\ Montpellier 2, France, \\ (2) Dynamique Moléculaire des Interactions Membranaires, UMR CNRS 5539, \\ Université Montpellier 2, France.
}

\section{Introduction}

The objective of this work is to propose a mechanical model and a numerical method devoted to the cytoskeleton (CSK) form-finding resulting from its structuration during cell spreading. It is now well assumed that the cell mechanics closely depends on its CSK architecture and on the tension and compression forces carried by its filaments. Several structural models have been therefore developed, especially those based on the tensegrity analogy. However, the observed topological and geometrical complexities of the CSK networks lead to difficulties for determining and using a realistic structure [Baudriller et al., 2006]. The presented method allows calculating such a complex architecture and the associated forces in the different CSK filaments of an adherent cell.

\section{CSK modelling and calculation}

A cell is modelled by using a granular medium with rigid grains representing respectively: (i) the nucleus, (ii) the centrosome, (iii) the CSK crosslinkers and (iv) some adhesion proteins like integrins. The grains are connected by at-distance mechanical interaction forces associated to the CSK filaments. These forces are initially tacit and the spreading process is then simulated by considering the growing of microtubules from the centrosome towards the integrins. These connect to the substratum by considering adhesive reactions. In the process, the microtubule growing pushes the other grains, thus changing the whole cell shape and generating interaction forces (Fig. 1). This result in a 3D network of compression forces and in an associated network of traction forces, which ensure the mechanical equilibrium of the overall structure connected to the substratum.

\section{Results}

The simulations lead to realistic compressive microtubule (star-like organization; Fig. 2) and associated tensile actin networks (Fig. 3). The results highlight basal higher tensions while the less ones appear on the apical face, consistently with in vivo distribution of strong stress fibres and of thin actin cortex.

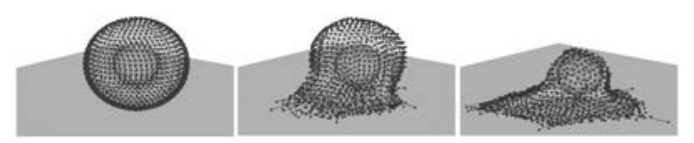

Figure 1: Steps of the simulation of cell spreading.

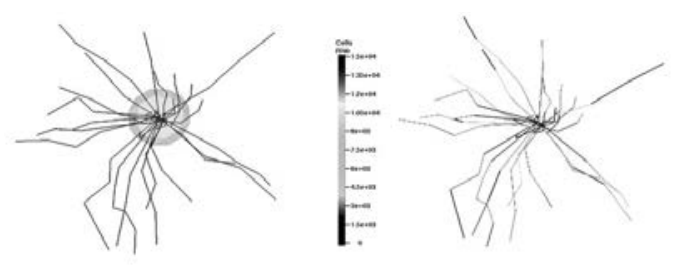

Figure 2: Obtained microtubule-like compressive network.

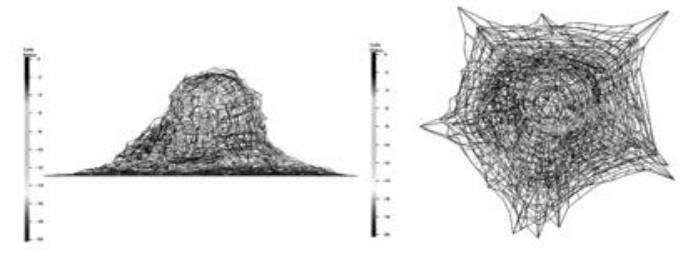

Figure 3: Resulting actin-like tensile network.

\section{Discussion and conclusion}

The presented model allows determining a complex and realistic 3D structure of the CSK filaments. Their internal tension and compression forces are mechanically equilibrated, such as considered in the tensegrity analogy. This approach may provide significant information for developing a mechanical model of the CSK structure and its spatial distribution of internal forces, which could be useful in further mechanical studies on adherent cell.

\section{References}

Baudriller et al., CRAS, 2006. 